Obere Extremität 2021 $\cdot 16: 114-119$ https://doi.org/10.1007/s11678-021-00641-0 Received: 23 October 2020

Accepted: 29 March 2021

Published online: 27 April 2021

(c) The Author(s) 2021

\author{
Christoph Lücke ${ }^{1,2}$ (D) $\cdot$ Marc Schnetzke ${ }^{1,2}$ Jennifer Engelke ${ }^{2}$ Janis Storg ${ }^{2}$. \\ Markus Loew' ${ }^{1}$ Sven Lichtenberg' \\ 'ATOS Clinic Heidelberg, German Joint Center Heidelberg, Heidelberg, Germany \\ ${ }^{2}$ BG Trauma Center Ludwigshafen, Clinic for Trauma and Orthopaedic Surgery at the University of \\ Heidelberg, Ludwigshafen on the Rhine, Germany
}

\title{
Correction of the CSA does not decrease the re-tear rate after SSP repair
}

\section{Results of a retrospective comparative study}

\section{Numerous risk factors determine the re-tear rate after arthroscopic rotator cuff repair. Risk factors such as age, tear size, and fatty infiltration are immutable by the surgical technique. Surgical reduction of the critical shoulder angle, which is also a risk factor for a re-tear of the supraspinatus tendon, was evaluated in a retrospective comparative study.}

\section{Background}

The re-tear rate after arthroscopic rotator cuff repair is high despite advances in surgical techniques [2, 19, 23]. In particular, age, tear size, retraction of the tendon, and fatty infiltration of the muscle determine the outcome after arthroscopic rotator cuff repair [5, 13, 24]. Recently, the critical shoulder angle (CSA) was described as a risk factor for both a rotator cuff tear as well as a re-tear after arthroscopic rotator cuff repair [7, 10, 16, 22].

The CSA was initially characterized by Moor et al. [16]. It describes the angle between the line of the lateral superior and inferior bony landmarks of the glenoid and the line between the lateral margin of the acromion and the inferior rim of the glenoid in a true anteroposterior radiograph (๑ Fig. 1). Moor et al. showed that patients with a CSA $>35^{\circ}$ have a greater risk for a supraspinatus tendon (SSP) tear.

A lower CSA $\left(<30^{\circ}\right)$ was associated with a greater risk for osteoarthritis [16].
Further research has found a connection between the CSA and rotator cuff tears, in particular with an SSP tear $[4,17$, 18]. Biomechanical research indicates that a higher CSA could lead to destabilizing joint forces during thoracohumeral abduction.

An increased SSP load may compensate the destabilization and the higher shear forces [9], which can result in more stress for the SSP.

There is debate regarding the optimal treatment of the CSA $[6,8,11,22]$. Questions are how to treat a high CSA, whether the CSA needs to be surgically corrected, if this correction changes the biomechanics for the benefit of the SSP, and, most importantly from a patient's point of view, whether the clinical and radiological outcome can be improved by changing the CSA.

The purpose of this study was to evaluate the effect of an additional lateral acromioplasty (ALAP) on postoperative CSA, tendon integrity, and clinical outcome after arthroscopic rotator cuff repair.

Our hypotheses were that: (1) ALAP decreases the CSA, (2) ALAP increases tear integrity, and (3) ALAP leads to better clinical outcome measured by the Constant Score (CS).

\section{Methods \\ Study design and patients}

A total of 48 patients with 49 treated SSPs were included after approval of the institutional ethics committee (No. 02/17). One patient had both shoulders treated for an SSP tear within 5 months. Patients with a CSA $>35^{\circ}$, a mechanical outlet impingement, and a rotator cuff tear were divided into two groups depending on their treating surgeon (S.L., M.L.). Inclusion criteria were age $<70$ years, no comorbidities (diabetes, rheumatoid predisorder, neurological disorders), no previous shoulder surgery on the same side, no osteoarthritis of the shoulder, atrophy of the SSP muscle<stage III, and written informed consent [26]. All patients had a lesion of the SSP. In total, 15 patients had an additional partial tear of the infraspinatus tendon and seven patients had an additional partial tear of the subscapularis tendon. The preoperative diagnostics included magnetic resonance imaging (MRI), evaluation of the CS, strength measurement in $90^{\circ}$ abduction and $30^{\circ}$ horizontal flexion, and a true anteroposterior radiograph.

The CSA was calculated from a true anteroposterior radiograph using the technique described by Moor et al. [16].

\section{Surgical technique}

Arthroscopic rotator cuff repair with subacromial decompression was performed 


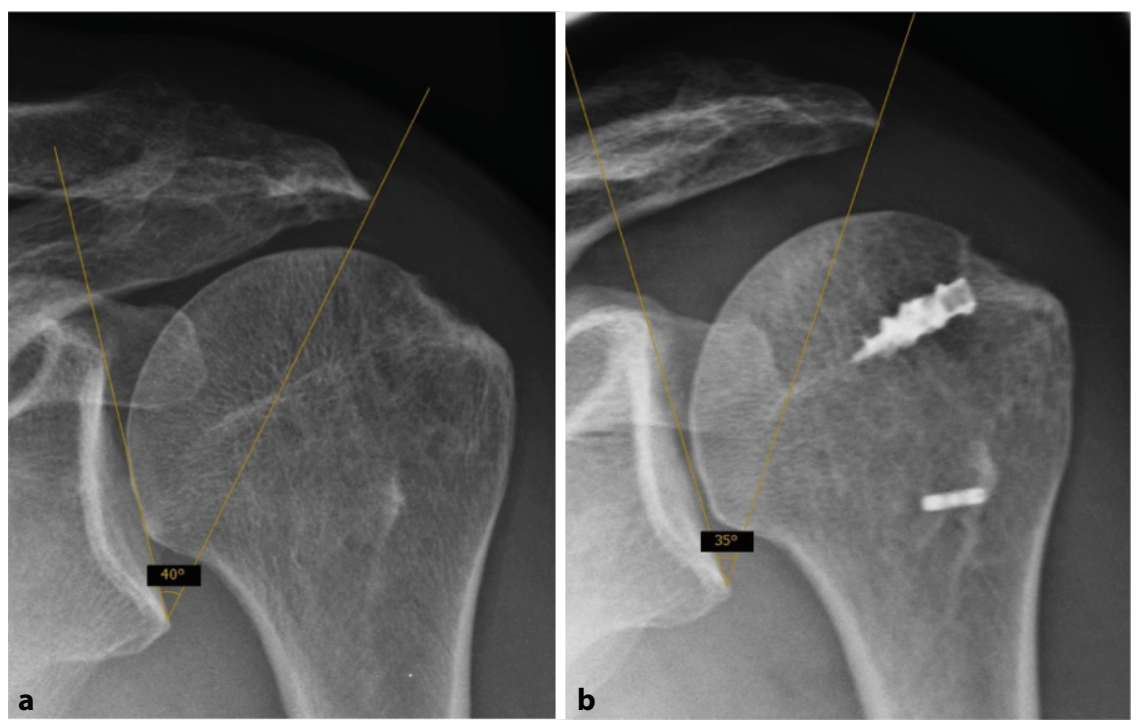

Fig. $1 \Delta$ Pre- $\mathbf{a}$ and postoperative $\mathbf{b}$ true anteroposterior radiographs of a left shoulder after an additional lateral acromioplasty with two Corkscrews and a proximal tenodesis button; $\mathrm{CCSA}=40^{\circ}$, b CSA $=35^{\circ}$

by one of the two senior surgeons. The 25 patients of group I received an ALAP to reduce the CSA, while the 23 patients (24 shoulders) of group II received standard acromioplasty. An ALAP was used to reduce the CSA by a $10-\mathrm{mm}$ arthroscopic resection of the lateral acromion. Other pathologies such as tendinitis of the long biceps tendon, SLAP lesion, subscapularis partial tear, or infraspinatus partial tear were repaired if required.

All patients received standardized physiotherapy. There were two rehabilitation protocols. The shoulder was immobilized in an abduction cushion $\left(30^{\circ}\right.$ abduction) for 3 weeks in the case of a single tendon reconstruction. If more than a single tendon repair was performed, immobilization duration was 6 weeks. For the first 6 weeks, only passive motion was allowed. Afterwards, active exercising was possible.

\section{Postoperative evaluation}

Within 2 days of surgery, a true anteroposterior radiograph was obtained to evaluate the CSA and the positions of the anchors. The follow-up to evaluate the effects of the ALAP took place at the earliest after 12 months. The CS, strength measurement, and the range of motion were used for clinical evaluation. Tendon integrity was evaluated by MRI using the classification by Sugaya [23]. Three patients were evaluated with ultrasound due to claustrophobia. The MRI and ultrasound examinations were assessed by the three senior surgeons (S.L., M.L., M.S.).

\section{Statistical analysis}

Prism 8 for macOS (Version 8.4.3, GraphPad Software, San Diego, CA USA) was used for statistical analysis. Mean and standard deviation (SD) were used to summarize the data. To evaluate the preand postoperative differences (CSA, CS) in the same group, a Wilcoxon matchedpairs signed rank test was used. A Mann-Whitney $U$ test was performed to evaluate differences in age, follow-up time, CSA, and CS between the groups and for the comparison in postoperative strength, pain, CS, and CSA between patients with healed and non-healed tendons. A two-tailed $p$ value of $<0.05$ was used to assess a possible significant difference. To evaluate the difference in healing rates of the two groups, Fischer's exact test was performed. Multiple logistic regression analysis was used to determine whether there are associations between tendon integrity and age, tear size, postoperative CSA, follow-up time, or group assignment. The significance level was set at $p<0.05$.

\section{Results}

\section{Clinical and radiological results of group I}

Mean follow-up time after surgery was 15.4 months \pm 5.5 (95\% CI: 13.1-17.7). Preoperative mean CSA was $38.0^{\circ} \pm 1.7$ (95\% CI: 37.3-38.7). Postoperative mean CSA was $34.48^{\circ} \pm 1.9$ (95\% CI: 33.7-35.3). The CSA significantly decreased from the preoperative to postoperative period ( $p<0.0001$, - Table 1$)$. Mean preoperative CS was $53.9 \pm 18.7$ (95\% CI: 46.2-61.6). Postoperatively, the mean CS significantly improved to 84.6 5 7.1 (95\% CI: 81.5-87.5; $p<0.0001$, - Table 1). The evaluation of tendon integrity by MRI and ultrasound showed 20 healed tendons (Sugaya 1-3) and five re-tears of the SSP (Sugaya 4-5).

\section{Clinical and radiological results of group II}

Mean follow-up time after surgery was 16.4 months \pm 5.2 (95\% CI: 14.2-18.6). Preoperative mean CSA was $37.8^{\circ} \pm 1.8$ (95\% CI: 37.0-38.5). After surgery, mean CSA was $37.6^{\circ} \pm 1.8$ (95\% CI: $36.8-38.4$ ). No significant difference between preoperative and postoperative CSAs was observed ( $p=0.125$, - Table 1). Mean preoperative CS was $48.9 \pm 19.5$ (95\% CI: 40.5-57.3). Postoperative mean CS significantly improved to $84.5 \pm 12.0$ (95\% CI: $79.4-89.7 ; p<0.0001$, - Table 1$)$. The evaluation of tendon integrity by MRI showed 20 healed tendons (Sugaya type I-III) and four re-tears of the SSP (Sugaya type IV-V).

\section{Group comparison}

Preoperatively, there was no significant difference in CSA $(p=0.6442)$ and CS $(p=0.6442)$ between both groups. There was a significant difference in age $(p=0.0038$, $\bullet$ Fig. 2$)$ : Patients in group I (mean age 62.1 years \pm 5.5 , 95\% CI: 59.9-64.4) were on average significantly older than those in group II (mean age 56.3 years $\pm 7.1,95 \%$ CI: 53.2-59.2).

Postoperatively, there was no significant difference in follow-up time $(p=0.9163$, • Fig. 2$)$ and CS ( $p=0.4557$, 
Obere Extremität 2021 · 16:114-119 https://doi.org/10.1007/s11678-021-00641-0

(c) The Author(s) 2021

C. Lücke · M. Schnetzke · J. Engelke · J. Storg · M. Loew · S. Lichtenberg

\section{Correction of the CSA does not decrease the re-tear rate after SSP repair. Results of a retrospective comparative study}

\section{Abstract}

Background. The re-tear rate after supraspinatus tendon (SSP) repair is high despite optimization of surgical techniques. The critical shoulder angle $(\mathrm{CSA})>35^{\circ}$ has been identified as a risk for re-tear. Surgical reduction of the CSA performing additional lateral acromioplasty (ALAP) is intended to reduce the risk.

Objective. We aimed to evaluate the effect of ALAP on the re-tear rate in patients with a CSA $>35^{\circ}$ after SSP repair.

Patients and methods. The study included 49 shoulders fulfilling predefined inclusion criteria. The patients were assigned to two groups; group I (25 SSP) was additionally treated with an ALAP. In group II (24 SSP) standard acromioplasty was performed during reconstruction. Magnetic resonance imaging and a clinical examination (Constant Score; CS) were performed 12 months after surgery. Results. After 15 months group I showed a re-tear rate of $20 \%$, a decreased CSA (mean $38.0^{\circ}$ preoperatively vs. $34.5^{\circ}$ postoperatively) and an increase in CS (mean 53.9 vs. 84.6). After 16 months group II showed a re-tear rate of $16.7 \%$, no difference between preand postoperative $\operatorname{CSA}\left(37.8^{\circ}\right.$ vs. $\left.37.6^{\circ}\right)$, and an increase in CS (48.9 vs. 84.5). There was no difference between the groups in terms of re-tears and postoperative CS ( $p=0.4557)$, but there was a significant difference in postoperative CSA $(p<0.0001)$. We did not find a decrease in the failure rate after ALAP. Unlike other studies, patients with a postoperative $\mathrm{CSA}>35^{\circ}$ did not have a worse clinical outcome and no increased rate of re-tears compared with patients with a mechanically reduced CSA.

Conclusion. Additive lateral acromioplasty does not decrease the re-tear rate after arthroscopic SSP repair. However, ALAP significantly decreases the CSA to normal values.

Keywords

Rotator cuff · Supraspinatus · Critical shoulder angle · Re-tear · Additional lateral acromioplasty

\section{Korrektur des CSA vermindert die Rerupturrate nach Rekonstruktion der Supraspinatussehne nicht. Ergebnisse einer retrospektiven Vergleichsstudie}

Zusammenfassung

Hintergrund. Die Rerupturrate nach Rekonstruktion der Supraspinatussehne (SSP) ist trotz Optimierung der Operationstechnik hoch. Der kritische Schulterwinkel $(\mathrm{CSA})>35^{\circ}$ gilt als Risiko für eine Reruptur. Die Verkleinerung des CSA mit einer additiven lateralen Akromioplastik (ALAP) soll das Risiko vermindern.

Ziel der Arbeit. Ziel war die Evaluation einer ALAP in einer retrospektiven Vergleichsstudie an Patienten mit einem $\mathrm{CSA}>35^{\circ}$ und einer Ruptur der SSP.

Material und Methoden. Es wurden 49 SSPRupturen, die vordefinierte Kriterien erfüllt haben, in die Studie eingeschlossen. Gruppe I (25 SSP) wurde zusätzlich mit einer ALAP behandelt. In Gruppe II (24 SSP) wurde keine ALAP bei der Rekonstruktion durchgeführt. Ab
12 Monate nach der Operation wurden eine Magnetresonanztomographie (MRT) und eine klinische Untersuchung (Constant Score, CS) durchgeführt.

Ergebnisse. Nach 15 Monaten zeigten sich in Gruppe I eine Rerupturrate von $20 \%$, eine signifikante Verkleinerung des CSA (durchschnittlich 38,0 vs. $34,5^{\circ}$ ) und Steigerung des CS (durchschnittlich 53,9 vs. 84,6 ). Nach 16 Monaten zeigten sich in Gruppe II eine Rerupturrate von 16,7\%, kein signifikanter Unterschied zwischen prä- und postoperativem CSA $\left(37,8\right.$ vs. $\left.37,6^{\circ}\right)$ und eine signifikante Steigerung des CS (48,9 vs. 84,5$)$. Zwischen den Gruppen bestand kein Unterschied in der Rerupturrate, im postoperativen CS $(p=0,4557)$, aber ein signifikanter Unterschied im postoperativen CSA $(p<0,0001)$.

Schlussfolgerung. Es konnte keine Verringerung der Rerupturrate durch eine ALAP nachgewiesen werden. Im Gegensatz zu anderen Studien wiesen Patienten mit einem postoperativen $\mathrm{CSA}>35^{\circ}$ kein schlechteres klinisches Ergebnis und keine erhöhte Rerupturrate im Vergleich zu Patienten mit verkleinertem CSA auf. Die ALAP führt zu einer signifikanten Verringerung des CSA zurück zu Normwerten.

Schlüsselwörter

Rotatorenmanschette · Supraspinatus . Kritischer Schulterwinkel · Reruptur · Additive laterale Akromioplastik
- Table 1, - Fig. 2). The postoperative CSA of patients in group I was significantly lower than that in group II $(p<0.0001$, • Table 1, • Fig. 2$)$.

No significant difference was found in re-tear rates between the two groups $(p>0.9999$, - Fig. 3$)$. There was no significant difference between patients with intact tendons and re-tears for postoperative strength $(p=0.5717)$, postoperative pain $(p=0.5733)$, postoperative
CS $(p=0.5916)$, and postoperative CSA $(p=0.5575$; $\bullet$ Table 2$)$.

Multiple logistic regression analysis to analyze the effect of various variables (explanatory variables) on the probability of a re-tear (tendon integrity) showed no significant effects for age, adjacent tear of infraspinatus and/or subscapularis tendon, postoperative CSA, follow-up time, or group assignment $(p>0.05)$.
No significant difference between patients with re-tears and patients with healed tendons was found for postoperative CSA $(p=0.5575)$, strength $(p=0.5717)$, pain $(p=0.5733)$, and CS $(p=0.5916$, ๑ Table 2$)$.

\section{Discussion}

The results suggest that an ALAP has no effect on the re-tear rate of the SSP after 


\begin{tabular}{|c|c|c|c|}
\hline & $\begin{array}{l}\text { Group I } \\
(n=25)\end{array}$ & $\begin{array}{l}\text { Group II } \\
(n=24)\end{array}$ & $\begin{array}{l}p \\
\text { (Group comparison) }\end{array}$ \\
\hline $\begin{array}{l}\text { CSA preop } \\
(95 \% \mathrm{Cl})\end{array}$ & $\begin{array}{l}38.0^{\circ} \pm 1.7^{\circ} \\
(37.3-38.7)\end{array}$ & $\begin{array}{l}37.8^{\circ} \pm 1.8^{\circ} \\
(37.0-38.5)\end{array}$ & 0.6442 \\
\hline $\begin{array}{l}\text { CSA postop } \\
(95 \% \mathrm{Cl})\end{array}$ & $\begin{array}{l}34.5^{\circ} \pm 1.9^{\circ} \\
(33.7-35.3)\end{array}$ & $\begin{array}{l}37.6^{\circ} \pm 1.8^{\circ} \\
(36.8-38.4)\end{array}$ & $<0.0001$ \\
\hline$p$ & $<0.0001$ & 0.125 & - \\
\hline $\begin{array}{l}\text { CS preop } \\
(95 \% \mathrm{CI})\end{array}$ & $\begin{array}{l}53.9 \pm 18.7 \\
(46.2-61.6)\end{array}$ & $\begin{array}{l}48.9 \pm 19.5 \\
(40.5-57.3)\end{array}$ & 0.3698 \\
\hline $\begin{array}{l}\text { CS postop } \\
(95 \% \mathrm{Cl})\end{array}$ & $\begin{array}{l}84.6 \pm 7.1 \\
(81.5-87.5)\end{array}$ & $\begin{array}{l}84.5 \pm 12.0 \\
(79.4-89.7)\end{array}$ & 0.4557 \\
\hline$p$ & $<0.0001$ & $<0.0001$ & - \\
\hline \multicolumn{4}{|c|}{$\begin{array}{l}\text { Values are given in mean } \pm \text { standard deviation } \\
\text { CSA critical shoulder angle, CS Constant Score, CI confidence interval, preop preoperatively, } \\
\text { postop postoperatively }\end{array}$} \\
\hline
\end{tabular}

\begin{tabular}{|c|c|c|c|}
\hline & $\begin{array}{l}\text { Intact tendons } \\
(n=40)\end{array}$ & $\begin{array}{l}\text { Re-tears } \\
(n=9)\end{array}$ & $p$ \\
\hline $\begin{array}{l}\text { Strength postop } \\
(95 \% \mathrm{Cl})\end{array}$ & $\begin{array}{l}7.2 \mathrm{~kg} \pm 3.0 \mathrm{~kg} \\
(6.2-8.2 \mathrm{~kg})\end{array}$ & $\begin{array}{l}6.3 \mathrm{~kg} \pm 2.1 \mathrm{~kg} \\
(4.7-7.9 \mathrm{~kg})\end{array}$ & 0.5717 \\
\hline $\begin{array}{l}\text { Pain postop, } \\
\text { NPRS } 0 \text { to } 15 \\
(95 \% \mathrm{Cl})\end{array}$ & $\begin{array}{l}0.9 \pm 1.6 \\
(0.4-1.4)\end{array}$ & $\begin{array}{l}0.4 \pm 0.7 \\
(-0.3-1.0)\end{array}$ & 0.5733 \\
\hline $\begin{array}{l}\text { CS postop } \\
(95 \% \mathrm{Cl})\end{array}$ & $\begin{array}{l}84.8 \pm 10.15 \\
(81.5-88.1)\end{array}$ & $\begin{array}{l}83.0 \pm 7.3 \\
(76.9-89.1)\end{array}$ & 0.5916 \\
\hline $\begin{array}{l}\text { CSA postop } \\
(95 \% \mathrm{Cl})\end{array}$ & $\begin{array}{l}35.9^{\circ} \pm 2.5^{\circ} \\
\left(35.12^{\circ}-36.7^{\circ}\right)\end{array}$ & $\begin{array}{l}36.3^{\circ} \pm 2.1^{\circ} \\
\left(34.8^{\circ}-37.9^{\circ}\right)\end{array}$ & 0.5575 \\
\hline \multicolumn{4}{|c|}{$\begin{array}{l}\text { Values are given in mean } \pm \text { standard deviation } \\
\text { CI confidence interval, postop postoperative CS Constant Score, NPRS Numeric Pain Rating Scale, } \\
\text { CSA critical shoulder angle }\end{array}$} \\
\hline
\end{tabular}

arthroscopic rotator cuff repair among patients with a CSA $>35^{\circ}$.

Recent studies reported re-tear rates between $15.3 \%$ and $28.5 \%$ [2, 3, 7, 19, 21]. Two of these studies evaluated the CSA and found an absence of tendon healing in $25 \%$ and $28.5 \%$ of their patients after rotator cuff repair and showed that patients with healed tendons had a lower CSA than patients with non-healed tendons. Subsequently, a higher CSA was categorized as a risk factor for re-tears [7, 21]. Further, a CSA $>35^{\circ}$ was described as a risk factor for SSP tears $[4,16]$. Since the CSA has been identified as a risk factor for tears and re-tears of the SSP, there are considerations to reduce the CSA by using ALAP $[1,8,12]$. Altintas et al. described no discontinuity in deltoid origin when performing the ALAP in a cadaveric in vitro study [1]. Further, Gerber et al. and Tauber et al. undertook a treatment study to show that ALAP does no
$18.4 \%$ is comparable to similar studies. Following the results, there is no recommendation for ALAP when performing an arthroscopic SSP repair on patients with a $\mathrm{CSA}>35^{\circ}$.

Overall, $20 \%$ of the patients treated with ALAP and $16.7 \%$ of the patients without surgical treatment of the CSA suffered a re-tear. In this collective, a higher postoperative CSA was not a risk factor for a re-tear. The control group in this study had a mean postoperative CSA slightly lower than $38^{\circ}$. Multiple logistic regression analysis showed no association between a higher postoperative CSA and tendon integrity. Patients with re-tears had equal results in terms of pain, strength, and CS in comparison with those with healed tendons at the follow-up. Besides not showing an effect on tendon integrity, ALAP did not improve the postoperative clinical outcome. These results are in contrast to the findings of previous studies on CSA $[7,8]$. Garcia et al. reported that patients with a higher CSA had a worse clinical outcome.

The strength in the group of patients with intact tendons was larger but there was no significant difference between the groups. This is in line with the findings of other studies on re-tear rates and risk factors [19, 24]. However, the present results are contradictory to other studies, which support the theory that ALAP has a positive effect on tendon integrity. A possible explanation for this might be the relatively short follow-up time used in the present study. The studies by Gerber et al. and by Garcia et al. had a mean follow-up of 30 and 26.1 months, respectively $[7,8]$. The mean overall followup of our study was only 15.9 months. Within this short time frame, ALAP did not show a positive effect. This is in line with the findings of Tashjian et al., who described a negative correlation between duration of follow-up with healed tendons at follow-up [24].

Due to the complex pathogenesis of a re-tear and various risk factors that influence the healing, the CSA might not have a strong impact on re-tears. Including more patients with radiological and clinical examinations will follow to 


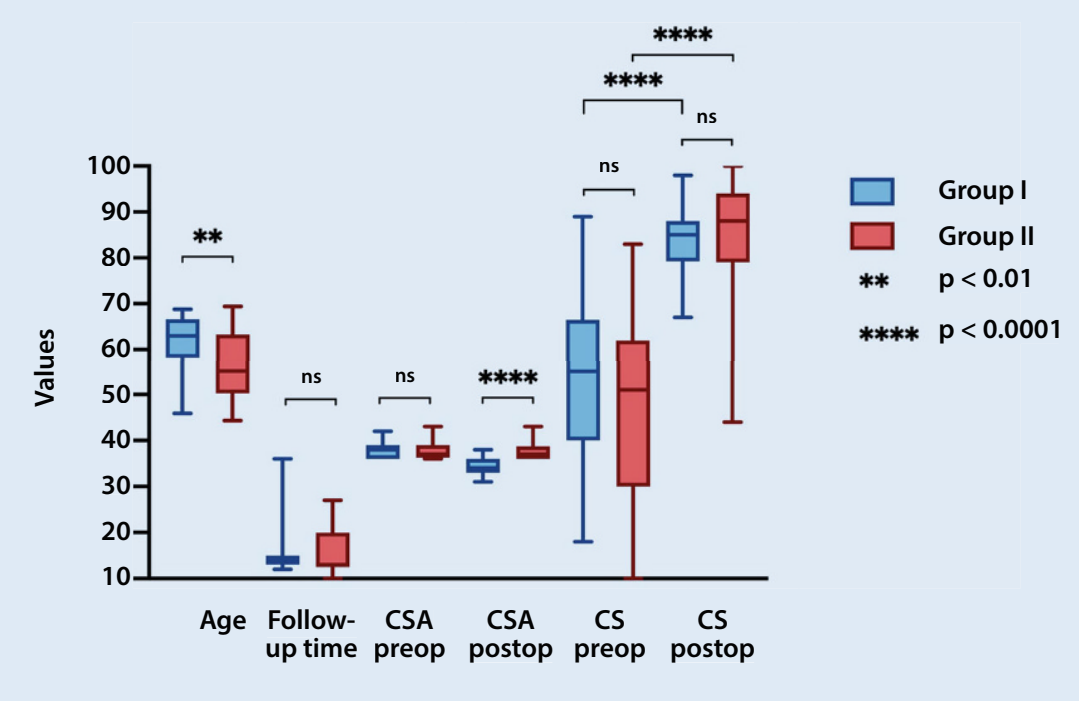

Fig. $2 \Delta$ Boxplot comparing the two groups in age, follow-up time, CSA, and CS. CSA critical shoulder angle, preop preoperative, postop postoperative, CS Constant score, ns not significant

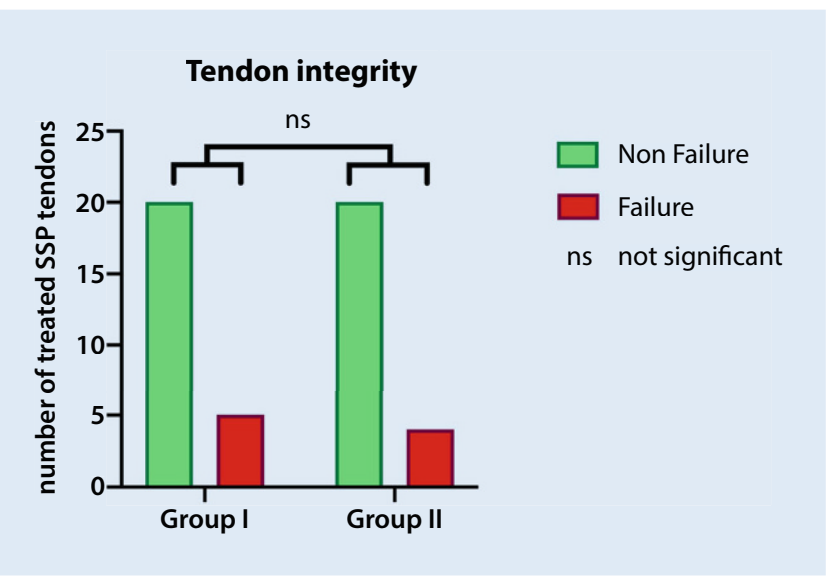

Fig. $3<$ Bar chart for comparison of the tendon integrity of groups I and II. SSP supraspinatus, ns not significant

Finally, the difference in tear size should also be mentioned. In group I, 15 patients had an isolated SSP tear and in group II, there were 12 isolated SSP tears. A multiple regression analysis that was performed to observe a possible effect of the tear size on the re-tear rate showed no significant association between tear size and tendon integrity.

\section{Practical conclusion}

- Additional lateral acromioplasty decreases the critical shoulder angle to standard values.

- No positive effect of additional lateral acromioplasty on the tendon integrity could be found.

- Additional lateral acromioplasty does not increase the clinical outcome as measured by the Constant Score.

\section{Corresponding address}

compensate for the lack of power in this study.

\section{Limitations}

One limitation of this study is the overall study size. Group samples of 25 and 24 patients are acceptable but there is a lack of power. The tear sizes were not matched, which can lead to bias in a small group. A larger sample size might have led to more significant effects between both the groups and the healing rates. As data collection is ongoing, further results will be published.

Furthermore, patients in group I (ALAP) were significantly older than those in group II. Patient age has also been described as a risk factor for a retear $[5,14,15,20]$. Multiple logistic re- gression analyzing a possible effect of age on the re-tear rate showed no association between age and tendon integrity.

The allocation of patients to the groups depended on the treating surgeon and was thereby not randomized. This results in a level of evidence of III.

There was only one independent surgeon who measured the CSA in the radiographs, which leads to potential subjective bias.

Another limitation is the short duration of follow-up, which might be correlated with a higher rate of healed tendons. This could be reversed by a longer follow-up [24]. Further studies will evaluate whether ALAP has a positive longterm effect on tendon healing in patients with a $\mathrm{CSA}>35^{\circ}$ after an arthroscopic SSP repair.

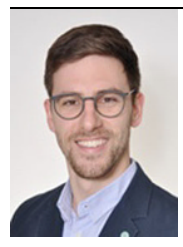

Christoph Lücke, MD

BG Trauma Center

Ludwigshafen, Clinic for

Trauma and Orthopaedic

Surgery at the University of

Heidelberg

Ludwig-Guttmann-Str. 13,

67071 Ludwigshafen on the Rhine, Germany chris.luecke@web.de

Funding. Open Access funding enabled and organized by Projekt DEAL.

\section{Declarations}

Conflict of interest. M. Loew is consultant for Wright Medical (Tornier) Cie. S. Lichtenberg is a paid consultant for Arthrex and Exactech and receives royalties from Arthrex. C. Lücke, M. Schnetzke, J. Engelke, and J. Storg declare that they have no competing interests.

All procedures followed were in accordance with the ethical standards of the responsible committee on human experimentation (institutional and national) and with the Helsinki Declaration of 1975 (in its most recently amended version). Informed consent was obtained from all patients included in the study.

Open Access. This article is licensed under a Creative Commons Attribution 4.0 International License, which permits use, sharing, adaptation, distribution and reproduction in any medium or format, as long as you give appropriate credit to the original author(s) and the source, provide a link to the Creative Commons licence, and indicate if changes were made. The images or other third party material in this article are included 
in the article's Creative Commons licence, unless indicated otherwise in a credit line to the material. If material is not included in the article's Creative Commons licence and your intended use is not permitted by statutory regulation or exceeds the permitted use, you will need to obtain permission directly from the copyright holder. To view a copy of this licence, visit http://creativecommons.org/licenses/by/4.0/.

\section{References}

1. Altintas B, Kaab M, Greiner S (2016) Arthroscopic lateral acromion resection (ALAR) optimizes rotator cuff tear relevant scapula parameters. Arch Orthop Trauma Surg 136:799-804

2. Anderson K, Boothby M, Aschenbrener D et al (2006) Outcome and structural integrity after arthroscopic rotator cuff repair using 2 rows of fixation: minimum 2-year follow-up. Am J Sports Med 34:1899-1905

3. Barth J, Andrieu K, Fotiadis E et al (2017) Critical period and risk factors for retear following arthroscopic repair of the rotator cuff. Knee Surg Sports Traumatol Arthrosc 25:2196-2204

4. Blonna D, Giani A, Bellato E et al (2016) Predominance of the critical shoulder angle in the pathogenesis of degenerative diseases of the shoulder. J Shoulder Elbow Surg 25:1328-1336

5. Boileau P, Brassart N, Watkinson DJ et al (2005) Arthroscopic repair of full-thickness tears of the supraspinatus: Does the tendon really heal? JBone Joint Surg Am 87:1229-1240

6. Chalmers PN, Beck L, Miller M et al (2020) Acromial morphology is not associated with rotator cuff tearing or repair healing. J Shoulder Elbow Surg 29(11):2229-2239

7. Garcia GH, Liu JN, Degen RM et al (2017) Higher critical shoulder angle increases the risk of retear after rotator cuff repair. J Shoulder Elbow Surg 26:241-245

8. Gerber C, Catanzaro S, Betz M et al (2018) Arthroscopic correction of the critical shoulder angle through lateral acromioplasty: a safe adjunct to rotator cuff repair. Arthroscopy 34:771-780

9. Gerber C, Snedeker JG, Baumgartner D et al (2014) Supraspinatus tendon load during abduction is dependent on the size of the critical shoulder angle: a biomechanical analysis. J Orthop Res 32:952-957

10. Heuberer PR, Plachel F, Willinger L et al (2017) Critical shoulder angle combined with age predict five shoulder pathologies: a retrospective analysis of 1000 cases. BMC Musculoskelet Disord 18:259

11. Kaiser D, Bachmann E, Gerber C et al (2018) Influence of the site of acromioplasty on reduction of the critical shoulder angle (CSA) - an anatomical study. BMCMusculoskelet Disord 19:371

12. Katthagen JC, Marchetti DC, Tahal DS et al (2016) The effects of arthroscopic lateral acromioplasty on the critical shoulder angle and the anterolateral deltoid origin: an anatomic cadaveric study. Arthroscopy 32:569-575

13. Kim YK, Jung KH, Kim JW et al (2018) Factors affecting rotator cuff integrity after arthroscopic repair for medium-sized or larger cuff tears: a retrospective cohort study. J Shoulder Elbow Surg 27:1012-1020

14. Le BT, Wu XL, Lam PHetal (2014) Factors predicting rotator cuff retears: an analysis of 1000 consecutive rotator cuffrepairs. Am JSports Med 42:1134-1142

15. Lee YS, Jeong JY, Park CD et al (2017) Evaluation of the risk factors for a rotator cuff retear after repair surgery. Am J Sports Med 45:1755-1761
16. Moor BK, Bouaicha S, Rothenfluh DA et al (2013) Is there an association between the individual anatomy of the scapula and the development of rotator cuff tears or osteoarthritis of the glenohumeral joint?: A radiological study of the critical shoulder angle. Bone Joint J95-B:935-941

17. Moor BK, Röthlisberger M, Müller DA et al (2014) Age, trauma and the critical shoulder angle accurately predict supraspinatus tendon tears. Orthop Traumatol Surg Res 100:489-494

18. Moor BK, Wieser K, Slankamenac K et al (2014) Relationship of individual scapular anatomy and degenerative rotator cuff tears. J Shoulder Elbow Surg 23:536-541

19. Neyton L, Godeneche A, Nove-Josserand L et al (2013) Arthroscopic suture-bridge repair for smal to medium size supraspinatus tear: healing rate and retear pattern. Arthroscopy 29:10-17

20. Rashid MS, Cooper C, Cook J et al (2017) Increasing age and tear size reduce rotator cuff repair healing rate at 1 year. Acta Orthop 88:606-611

21. Scheiderer B, Imhoff FB, Johnson JD et al (2018) Higher critical shoulder angle and acromion index are associated with increased retear risk after isolated supraspinatus tendon repair at short-term follow up. Arthroscopy 34:2748-2754

22. Sheean AJ, Sa D, Woolnough T et al (2019) Does an increased critical shoulder angle affect re-tear rates and clinical outcomes following primary rotator cuff repair? A systematic review. Arthroscopy 35:2938-2947.e1

23. Sugaya H, Maeda K, Matsuki K et al (2007) Repair integrity and functional outcome after arthroscopic double-row rotator cuff repair. A prospective outcome study. J Bone Joint Surg Am 89:953-960

24. Tashjian RZ, Hollins AM, Kim HM et al (2010) Factors affecting healing rates after arthroscopic double-row rotator cuff repair. Am J Sports Med 38:2435-2442

25. Tauber M, Habermeyer P, Zumbansen N et al (2020) Lateral acromioplasty for correction of the critical shoulder angle. Obere Extrem 15:228-232

26. Thomazeau H, Rolland Y, Lucas C et al (1996) Atrophy of the supraspinatus belly. Assessment by MRI in 55 patients with rotator cuff pathology. Acta OrthopScand 67:264-268 